\title{
Combination therapy of infliximab and thalidomide for refractory entero-Behcet's disease: a case report
}

Yue $\mathrm{Li}^{1+}$, Zelong Han ${ }^{1 \dagger}$, Xianfei Wang ${ }^{1,2}$, Zhihui Mo ${ }^{1}$, Wei Zhang ${ }^{1}$, Aimin $\mathrm{Li}^{1}$ and Side Liu*

\begin{abstract}
Background: Behcet's disease (BD) is a systemic inflammatory disease with the histopathological features of leukocytoclastic vasculitis that affects nearly all organs and systems. When it involves the intestine, it is called entero-Behcet's disease (entero-BD).
\end{abstract}

Case presentation: Here we described a 23-year-old man with entero-BD refractory to conventional therapies who responded well to the combination therapy of infliximab, an anti-tumor-necrosis-factor (TNF)-alpha antibody, and thalidomide. After combination treatment, the patient's symptoms improved greatly and his Crohn's Disease Activity Index (CDAl) score decreased from 344 to 52 points, accompanied by a body weight increase from $53 \mathrm{~kg}$ to $64 \mathrm{~kg}$. A follow-up endoscopy performed 10 weeks after the treatment showed significant improvement and the patient's multiple ulcers had healed well.

Conclusion: The combination therapy of infliximab and thalidomide appears to be clinically effective in a patient with refractory entero-BD. However, further studies need to be performed to evaluate the efficacy of this combination therapy.

Keywords: Entero-Behcet's disease, Infliximab, Thalidomide

\section{Background}

Behcet's disease $(\mathrm{BD})$ is a chronic relapsing vasculitis characterized by recurrent oral and genital ulcerations with uveitis and is more prevalent around countries along the ancient Silk Route [1]. Entero-Behcet's disease (entero-BD) is characterized by intestinal inflammation with round and oval ulcers typically in the ileocaecum and is associated with gastrointestinal symptoms, which are often uncontrollable, relapsing, and can cause acute intestinal bleeding or perforation [2,3]. Gastrointestinal involvement has been reported in $3 \%-26 \%$ of patients with $\mathrm{BD}$ [4]. The etiology of $\mathrm{BD}$ is still unknown, but tissue damage that occurs in $\mathrm{BD}$ patients is believed to be caused by oxygen radicals, which are generated by proinflammatory cytokines and arachidonic acid metabolites

\footnotetext{
* Correspondence: liuside@163.com

${ }^{\dagger}$ Equal contributors

'Guangdong Provincial Key Laboratory of Gastroenterology, Department of Gastroenterology, Nanfang Hospital, Southern Medical University, Guangzhou 510515, China

Full list of author information is available at the end of the article
}

[5,6]. Although corticosteroids, 5-aminosalicylic acid derivatives, immunosuppressive agents, and immunomodulators have been used to treat BD patients with varying degrees of success, $\mathrm{BD}$ is associated with severe morbidity and considerable mortality [7]. Tumor necrosis factor (TNF)-alpha plays an important role in this $\mathrm{T}$ helper cell type 1 (Th1)-mediated disease [8]. Infliximab, a monoclonal antibody to TNF-alpha, which neutralizes TNF-alpha and down-regulates the expression of granulocyte-macrophage colony-stimulating factor has been demonstrated to be an effective therapy for Crohn's disease, rheumatoid arthritis and other Th1-mediated disorders [9]. However, the single use of infliximab is not efficient in all BD patients [10,11]. Thalidomide selectively inhibits the production of TNF-alpha in monocytes and reduces its activity by a mechanism distinct from infliximab [12,13]. Many publications have reported the possible use of thalidomide for a wide range of conditions such as BD [14-17]. Here, we utilized a combination therapy of infliximab and thalidomide and showed that 
it appears to be clinically effective in a patient with refractory entero-BD.

\section{Case presentation}

A 23-year-old man was admitted to our hospital because he had recurrent abdominal pain and fever for more than 2 years. The patient began to have a burning pain in the epigastrium in October 2008, which mostly occurred at night and when he was hungry. The pain occurred once every 1 to 2 months, each time lasting for 1 to 2 days, accompanied by fever, with temperature fluctuating between $38-39^{\circ} \mathrm{C}$, which would alleviate by itself. The patient did not have diarrhea, night sweats or other symptoms. Laboratory examination in the local hospital revealed white blood cell (WBC) $10.0 \times 10^{9} / \mathrm{L}$ (normal 3.6-9.7 $\times$ $10^{9} / \mathrm{L}$ ), neutrophil rate $79.2 \%$ (normal $50-70 \%$ ), hemoglobin $116 \mathrm{~g} / \mathrm{L}$ (normal 120-160 g/L) and Creactive protein (CRP) $87.7 \mathrm{mg} / \mathrm{L}$ (normal $0-5 \mathrm{mg} / \mathrm{L}$ ). Erythrocyte sedimentation rate (ESR) was $27.0 \mathrm{~mm} / \mathrm{h}$ (normal 0-15 mm/h), and occult blood test (OBT) was positive. The patient was a non-smoker with no family history of inflammatory bowel disease. Gastroscopy revealed duodenal bulb ulcers. Although acid inhibitors and antipyretics were used in the local hospital, his symptoms did not improve. He was referred to our department for further evaluation. On admission, a physical examination found an enlarged submental lymph node which was soft and removable without pressing pain. After admission, laboratory examination indicated that WBC, OB, CRP, and ESR were normal. The patient tested negative for autoantibodies to nuclear antigen, double-stranded deoxyribonucleic acid, nuclear ribonucleoprotein, anti-saccharomces cerevisiae antibodies and anti-neutrophil cytoplasmic antibodies. In addition, Pathergy and Widal tests were both negative. Gastroscopy and double balloon enteroscopy revealed duodenal bulb ulcers and scattered round small ulcers in the jejunum with no evidence of Helicobacter Pylori infection (Figure 1A, 1B). Biopsy of a deeply ulcerated area of jejunum revealed nonspecific mucosal inflammation without granulomata (Figure 1C). Positron emission tomography/computed tomography (PET-CT) found multiple and flake concentration around the jejunum and ileum. Inflammatory and hyperplastic lymph nodes without increased metabolism were found in the abdominal, retroperitoneal and mesenteric region (Figure 2).

Although various test had been conducted, the diagnosis of the patient remained unresolved. His medical history was long and he did not complaint of hematochezia. PETCT scans did not reveal the presence of any abdominal mass nor any sign of neoplasma. Most significantly, the biopsy specimen from the deeply ulcerated area of the jejunum did not show the presence of lymphoma cells, therefore the possibility of intestinal lymphoma was excluded. Coeliac disease is more prevalent in Caucasian, but our patient is an Asian. Meanwhile, eating wheat foods does not have obvious relation with the patient's symptoms. Therefore, the possibility of coeliac disease was also excluded. However Crohn's disease, tuberculosis of the intestines and BD were difficult to differentiate from each other and remained possible causative conditions. After discharge, the patient still suffered from recurrent abdominal pain. He returned to our department with recurrent abdominal pain and fever in March 2012. On further investigation it was discovered that the patient had had recurrent oral ulcers, genital ulcers and occasional blurred vision since childhood. Capsule endoscopy was carried out and revealed multiple ulcers in the small intestine, mainly located in the jejunum (Figure 3A, 3B). BD is diagnosed based on clinical evaluation. Our patient was diagnosed with entero-BD based on the International Criteria for Behcet's Disease [18]. However, although he had been treated with prednisone, mesalazine, cyclophosphamide and colchicines in the local hospital, his symptoms had not improved. After discussion with the patient, a combination therapy including infliximab (5 mg/kg), thalidomide (100 mg,qd) and prednisone (15 mg/day) was prescribed. Infliximab was infused at weeks $0,2,6,14,22$ and 30 according to instructions. No medication-related adverse reactions were observed and

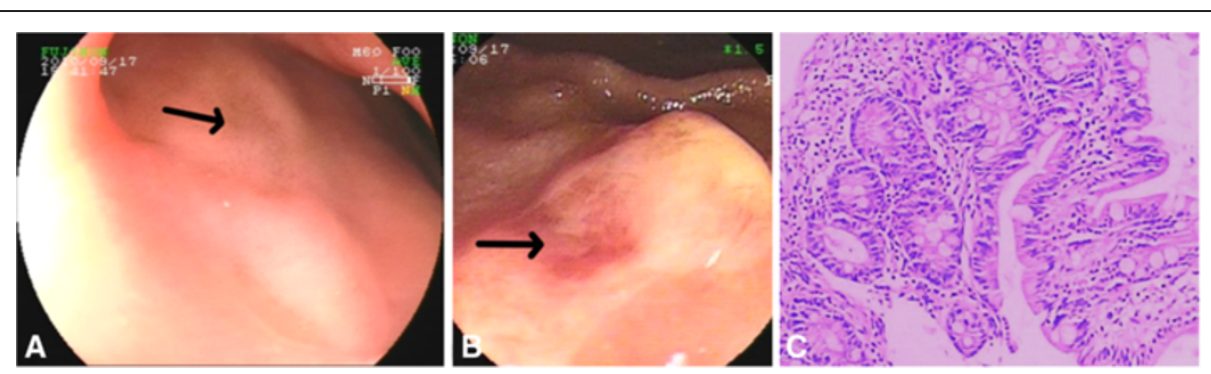

Figure 1 Related auxiliary examinations were conducted in order to make a definitive diagnosis. Gastroscopy revealed a scar (arrow) left on the anterior wall of the duodenum from a healed ulcer (A). Double balloon enteroscopy revealed a round ulcer (arrow) characterized by hyperemia and erosion (B). Biopsy specimens from the jejunum revealed chronic inflammatory infiltrate consisting of a mixture of neutrophils, lymphocytes and plasma cells (C). 


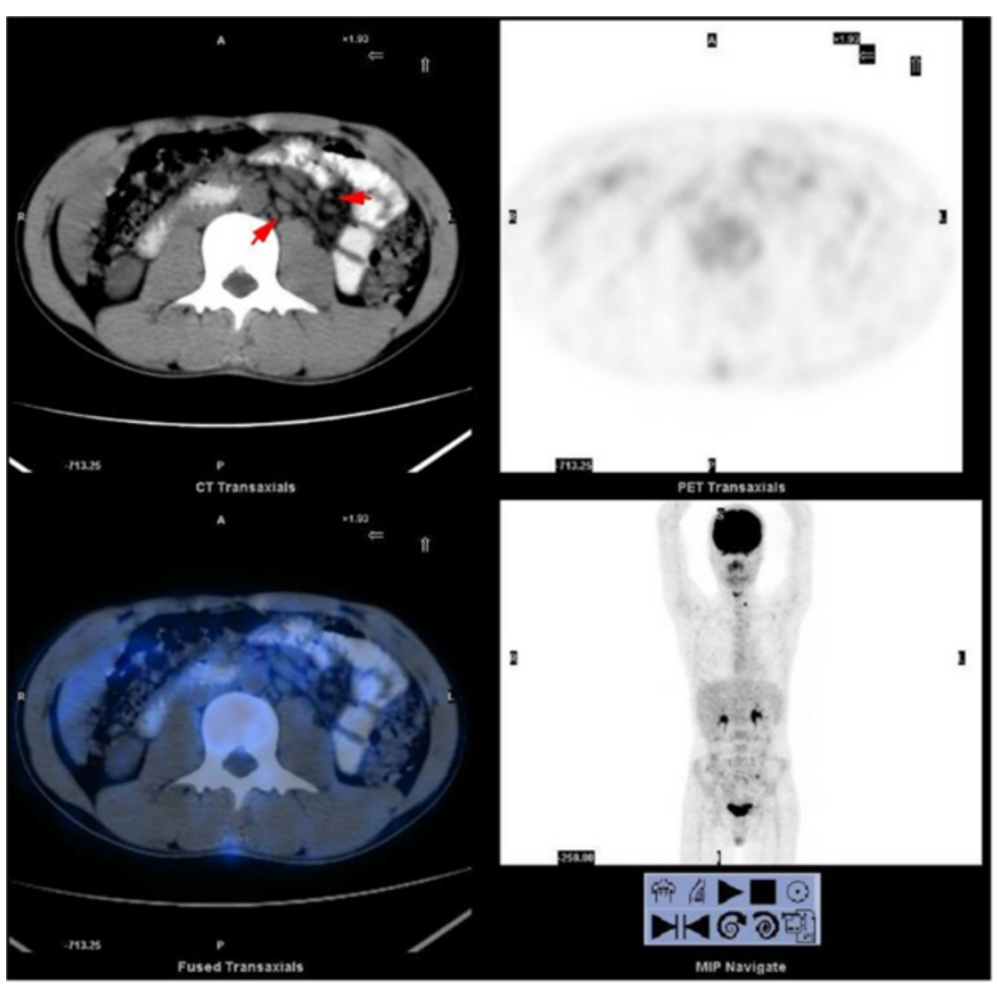

Figure 2 PET-CT revealed multiple enlarged lymph nodes (arrow) in the abdominal and mesenteric region without abnormal concentration of 18 F-fluorodeoxyglucose (18 F-FDG).

the prednisone dose was gradually tapered during the treatment. After the first infusion with infliximab the patient's abdominal pain and fever disappeared despite complete withdrawal of steroids. CRP decreased from $87.7 \mathrm{mg} / \mathrm{L}$ to $0.7 \mathrm{mg} / \mathrm{L}$ and ESR decreased from $27.0 \mathrm{~mm} / \mathrm{h}$ to $4 \mathrm{~mm} / \mathrm{h}$. Because most of his symptoms were gastrointestinal, the Crohn's Disease Activity Index (CDAI) was selected as an objective measure of response to the therapy. The CDAI score decreased from 344 to 52 points during therapeutic period (Figure 4). Meanwhile, his body weight increased from $53 \mathrm{~kg}$ to $64 \mathrm{~kg}$. Capsule endoscopy performed 10 weeks after the last infusion showed marked endoscopic improvement and the patient's multiple ulcers had healed well (Figure 3C, 3D).

\section{Discussion}

Although oral ulceration, genital ulceration and eye disease are classical triad symptoms of $\mathrm{BD}$, the cardiovascular, gastrointestinal, musculoskeletal and central nervous systems can also be affected [19]. The pathogenesis of BD remains unknown but major determinants involving genetic and immune system abnormalities have been recently reported [20]. The diagnosis of entero-BD is made according to the International Criteria for Behcet's Disease taking into account the patient's gastrointestinal symptoms and intestinal ulcers as detected by endoscopy. However, when the patient's symptoms are not typical, the diagnosis may be difficult. In intestinal $\mathrm{BD}$, deep ulcers develop in the gastrointestinal tract, typically in the ileocaecum. However, the patient presented in this report had scattered small ulcers which were mainly in the jejunum. In addition, the pathergy test was negative, which also complicated the diagnosis. After collecting a detailed medical history, the diagnosis was eventually established. In our experience, comprehensive inquiry and a detailed medical history is important in the diagnosis of entero-BD as well as endoscopic examination. When gastroscopic and colonoscopic investigations do not reveal significant pathological changes, double-balloon enteroscopy or capsule endoscopy may contribute to the detection of lesions since many entero-BD ulcers are atypically located in the ileum and jejunum. Additionally, the pathergy test is not always positive and the clinician should not be confused by the negative results when making a diagnosis of BD.

Entero-BD is often difficult to manage by conventional therapies such as corticosteroids, 5-aminosalicylic acid derivatives and immunosuppressive agents. Recently, offlabel use of anti-tumor necrosis factor agents for BD has increased, suggesting that TNF blockade represents an important therapeutic approach for patients with severe and resistant $\mathrm{BD}$, but randomized controlled trials are lacking [7,21-23]. Infliximab has been reported to have 


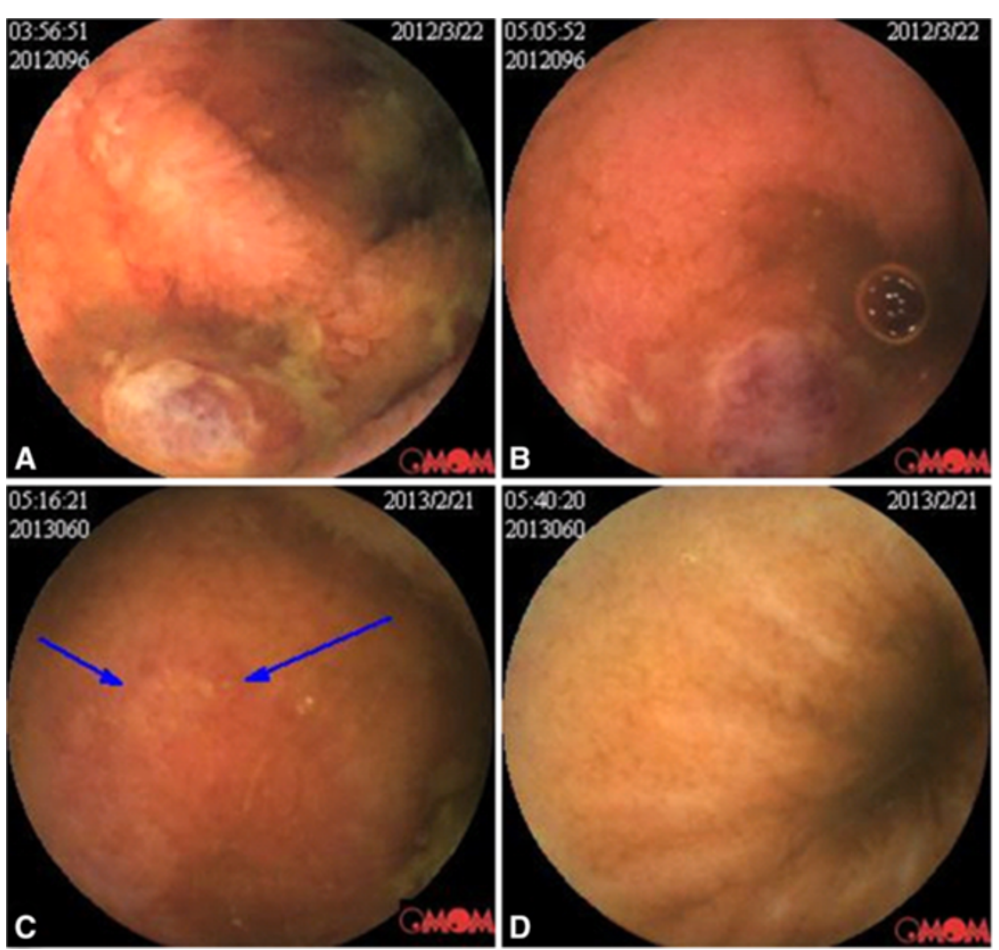

Figure 3 Before infusion, capsule endoscopy showed multiple oval ulcers with purplish red color change and thin fur attached on the surface. Congestion and edema of the mucosa can be seen around the ulcers which were widely distributed in the small intestine (A, B). Repeated capsule endoscopy showed improvement in ulcerations and inflammation 10 weeks after the last infusion (C, D). Most of the ulcers had healed, leaving a small sheet of erosion (arrow).

rapid and excellent efficacy in patients with refractory entero-BD [24-27]. Although 91\% of BD patients responded to infliximab, some patients responded poorly to the treatment of infliximab $[10,11,21]$. In our experience, entero-BD patients usually respond poorly to various conventional treatments such as prednisone, mesalazine, cyclophosphamide and colchicines, which were all used for the treatment of our patient in the local hospital. Since our patient failed to respond to various conventional treatments, along with the concern that he may respond poorly to a single use of infliximab, we decided to treat him with a combination therapy of infliximab and thalidomide, which may synergize due to a complete blockade of TNF- $\alpha$.

After the treatment, his symptoms and intestinal lesions improved without adverse effects. To our knowledge, this

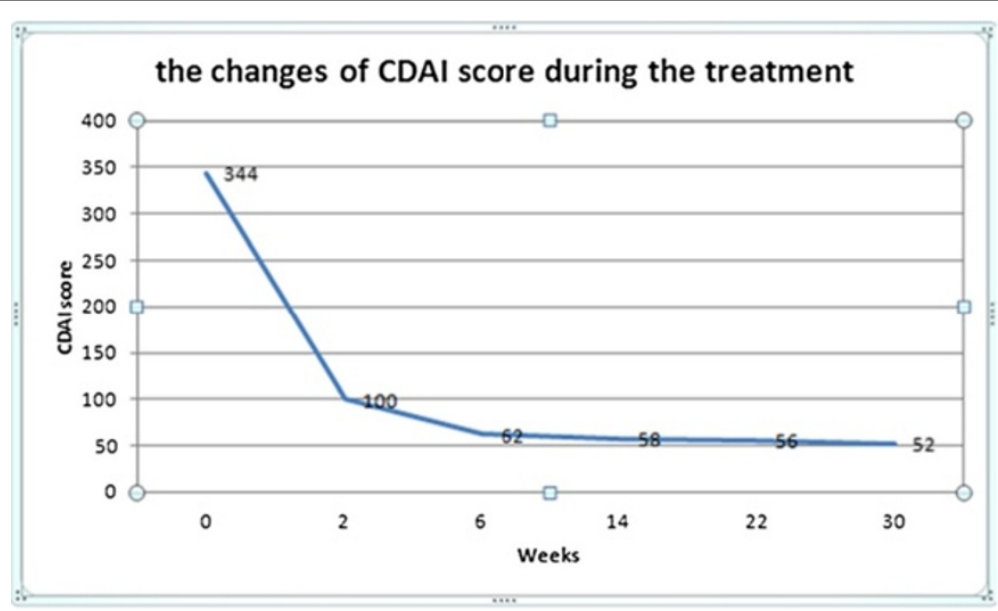

Figure 4 A CDAI score $<150$ points is considered clinical remission in a patient with CD. After the first infusion, the patient noticed great improvement in symptoms, and his CDAI score declined dramatically from 344 to 100 points, which indicated clinical remission. 
is the first report of a treatment regime using the combination therapy of infliximab and thalidomide. The good response of the patient to this combination therapy is likely a result of a complete TNF- $\alpha$ blockade by the two agents. Although our report describes only one case, the improvement in symptoms of the patient supports evidence that increased levels of TNF- $\alpha$ play a critical role in the inflammatory process associated with $\mathrm{BD}$. In view of limitations of the present treatment for intestinal BD, combination therapy with infliximab and thalidomide appears to be an effective approach for the treatment of entero-BD and perhaps other manifestations of BD.

\section{Conclusion}

Based on our report, the combination therapy of infliximab and thalidomide could be selected as an effective approach for the patients with refractory entero-BD. However, further studies need to be performed to evaluate the efficacy of this combination therapy.

\section{Consent statement}

Written informed consent was obtained from the patient for publication of this case report and any accompanying images. A copy of the written consent is available for review by the Editor of this journal.

\begin{abstract}
Abbreviations
BD: Behcet's disease; TNF: Tumor necrosis factor; WBC: White blood cell; CRP: C-reactive protein; ESR: Erythrocyte sedimentation rate; OB: Occult blood test; PET-CT: Positron emission tomography/computed tomography; CDAl: The Crohn's Disease Activity Index.
\end{abstract}

\section{Competing interests}

We declare that we have no competing interests.

\section{Authors' contributions}

$Y L$ and $\mathrm{ZH}$ contributed equally to this paper. $\mathrm{YL}$ designed and drafted the manuscript. ZH participated in the design of the study. ZM and XW performed the data collecting of this case. WZ and AL helped to revise the manuscript. SL conceived of the study, and participated in its design and coordination and helped to draft the manuscript. All authors read and approved the final manuscript.

\section{Acknowledgements}

Project was supported by Guangdong Province Universities and Colleges Pearl River Scholar Funded Scheme.

At the point of finishing this paper, l'd like to express my sincere thanks to all those who have provided support during the course of my writing of this paper. First of all, I'd like to take this opportunity to show my sincere gratitude to my supervisor, Professor Side Liu, who has given me so much useful advice on my writing, and has tried her best to improve my paper. Secondly, I'd like to express my gratitude to my classmates who offered me timely references and information. Last but not the least, I'd like to thank the patient, Mr. Guo, who has given his consent for the case report to be published.

\section{Author details}

${ }^{1}$ Guangdong Provincial Key Laboratory of Gastroenterology, Department of Gastroenterology, Nanfang Hospital, Southern Medical University, Guangzhou 510515, China. ${ }^{2}$ Department of Gastroenterology, Affiliated Hospital of North Sichuan Medical College, Nanchong, China.
Received: 20 July 2013 Accepted: 27 November 2013

Published: 9 December 2013

\section{References}

1. Keino H, Okada AA: Behcet's Disease: global epidemiology of an old silk road disease. Br J Ophthalmol 2007, 91(12):1573-1574.

2. Hisamatsu T, Kishikawa H, Fukuya H, Matsuoka K, Mizuno Y, Nishida J: Combination therapy including pentoxifylline for entero-Behcet's disease. Bull Tokyo Dent Coll 2001, 42(3):169-176.

3. Naganuma M, Sakuraba A, Hisamatsu T, Ochiai H, Hasegawa H, Ogata H, Iwao Y, Hibi T: Efficacy of infliximab for induction and maintenance of remission in intestinal Behcet's disease. Inflamm Bowel Dis 2008, 14(9):1259-1264.

4. Sakane T, Takeno M, Suzuki N, Inaba G: Behcet's disease. N Engl J Med 1999, 341(17):1284-1291.

5. Kitauchi S, Nishi S, Nishioka S: Intestinal Behcet's disease: epidemiology, pathophysiology and potential of mesalazine. BioDrugs 2000, 13(6):409-413.

6. Cho SB, Cho S, Bang D: New insights in the clinical understanding of Behcet's disease. Yonsei Med J 2012, 53(1):35-42.

7. Hassard PV, Binder SW, Nelson V, Vasiliauskas EA: Anti-tumor necrosis factor monoclonal antibody therapy for gastrointestinal Behcet's disease: a case report. Gastroenterology 2001, 120(4):995-999.

8. Ferrante A, Ciccia F, Principato A, Giardina AR, Impastato R, Peralta S, Triolo G: A Th1 but not a Th17 response is present in the gastrointestinal involvement of Behcet's disease. Clin Exp Rheumatol 2010, 28(4 Suppl 60):S27-S30.

9. Kirman I, Whelan RL, Nielsen OH: Infliximab: mechanism of action beyond TNF-alpha neutralization in inflammatory bowel disease. Eur $J$ Gastroenterol Hepatol 2004, 16(7):639-641.

10. Leccese P, D'Angelo S, Angela P, Coniglio G, Olivieri I: Switching to adalimumab is effective in a case of neuro-Behcet's disease refractory to infliximab. Clin Exp Rheumatol 2010, 28(4 Suppl 60):S102.

11. Shimizu Y, Takeda T, Matsumoto R, Yoshida K, Nakajima J, Atarashi T, Yanagisawa H, Kikuchi K, Kikuchi H: Clinical efficacy of adalimumab for a postoperative marginal ulcer in gastrointestinal Behcet disease. Nippon Shokakibyo Gakkai Zasshi 2012, 109(5):774-780.

12. Sampaio EP, Sarno EN, Galilly R, Cohn ZA, Kaplan G: Thalidomide selectively inhibits tumor necrosis factor alpha production by stimulated human monocytes. J Exp Med 1991, 173(3):699-703.

13. Calabrese L, Fleischer AB: Thalidomide: current and potential clinical applications. Am J Med 2000, 108(6):487-495.

14. Hamuryudan V, Mat C, Saip S, Ozyazgan Y, Siva A, Yurdakul S, Zwingenberger $\mathrm{K}$, Yazici $\mathrm{H}$ : Thalidomide in the treatment of the mucocutaneous lesions of the Behcet syndrome. A randomized, doubleblind, placebo-controlled trial. Ann Intern Med 1998, 128(6):443-450.

15. Shek LP, Lee YS, Lee BW, Lehman TJ: Thalidomide responsiveness in an infant with Behcet's syndrome. Pediatrics 1999, 103(6 Pt 1):1295-1297.

16. Kari JA, Shah V, Dillon MJ: Behcet's disease in UK children: clinical features and treatment including thalidomide. Rheumatology (Oxford) 2001, 40(8):933-938.

17. Yasui K, Uchida N, Akazawa Y, Nakamura S, Minami I, Amano Y, Yamazaki T: Thalidomide for treatment of intestinal involvement of juvenile-onset Behcet disease. Inflamm Bowel Dis 2008, 14(3):396-400.

18. Criteria for diagnosis of Behcet's disease: International study group for Behcet's disease. Lancet 1990, 335(8697):1078-1080.

19. Ambrose NL, Haskard DO: Differential diagnosis and management of Behcet syndrome. Nat Rev Rheumatol 2013, 9(2):79-89.

20. Pineton De Chambrun M, Wechsler B, Geri G, Cacoub P, Saadoun D: New insights into the pathogenesis of Behcet's disease. Autoimmun Rev 2012, 11(10):687-698.

21. Arida A, Fragiadaki K, Giavri E, Sfikakis PP: Anti-TNF agents for Behcet's disease: analysis of published data on 369 patients. Semin Arthritis Rheum 2011, 41(1):61-70.

22. Iwata S, Saito K, Yamaoka K, Tsujimura S, Nawata M, Suzuki K, Tanaka Y: Effects of anti-TNF-alpha antibody infliximab in refractory entero-Behcet's disease. Rheumatology (Oxford) 2009, 48(8):1012-1013.

23. Benitah NR, Sobrin L, Papaliodis GN: The use of biologic agents in the treatment of ocular manifestations of Behcet's disease. Semin Ophthalmol 2011, 26(4-5):295-303. 
24. Travis SP, Czajkowski M, McGovern DP, Watson RG, Bell AL: Treatment of intestinal Behcet's syndrome with chimeric tumour necrosis factor alpha antibody. Gut 2001, 49(5):725-728.

25. Kram MT, May LD, Goodman S, Molinas S: Behcet's ileocolitis: successful treatment with tumor necrosis factor-alpha antibody (infliximab) therapy: report of a case. Dis Colon Rectum 2003, 46(1):118-121.

26. Ju JH, Kwok SK, Seo SH, Yoon CH, Kim HY, Park SH: Successful treatment of life-threatening intestinal ulcer in Behcet's disease with infliximab: rapid healing of Behcet's ulcer with infliximab. Clin Rheumatol 2007, 26(8):1383-1385

27. Lee JH, Kim TN, Choi ST, Jang BI, Shin KC, Lee SB, Shim YR: Remission of intestinal Behcet's disease treated with anti-tumor necrosis factor alpha monoclonal antibody (Infliximab). Korean J Intern Med 2007, 22(1):24-27.

doi:10.1186/1471-230X-13-167

Cite this article as: Li et al: Combination therapy of infliximab and thalidomide for refractory entero-Behcet's disease: a case report. BMC Gastroenterology 2013 13:167.

\section{Submit your next manuscript to BioMed Central and take full advantage of:}

- Convenient online submission

- Thorough peer review

- No space constraints or color figure charges

- Immediate publication on acceptance

- Inclusion in PubMed, CAS, Scopus and Google Scholar

- Research which is freely available for redistribution 